\title{
Correction to: Virtual, Augmented and Mixed Reality. Industrial and Everyday Life Applications
}

Jessie Y. C. Chen and Gino Fragomeni

\author{
Correction to: \\ J. Y. C. Chen and G. Fragomeni (Eds.): \\ Virtual, Augmented and Mixed Reality, LNCS 12191, \\ https://doi.org/10.1007/978-3-030-49698-2
}

The original version of this chapter 2 was revised. A video was added to help provide clarity and a visual explanation of the paper.

The title of the originally published chapter 27 contained a typo. The title was corrected.

The updated version of these chapters can be found at https://doi.org/10.1007/978-3-030-49698-2_2

https://doi.org/10.1007/978-3-030-49698-2_27

(C) Springer Nature Switzerland AG 2020

J. Y. C. Chen and G. Fragomeni (Eds.): HCII 2020, LNCS 12191, p. C1, 2020.

https://doi.org/10.1007/978-3-030-49698-2_29 